\title{
VALENCE-BAND PHOTOEMISSION INTENSITIES IN THORIUM DIOXIDE
}

\author{
W.P. Ellis*, A.M. Boring*, J.W. Allen ${ }^{\dagger}$, L.E. Cox*, R.D. Cowan*, B.B. Pate ${ }^{\ddagger}$, A.J. Arko* and I. Lindau ${ }^{\ddagger}$ \\ *University of California, Los Alamos National Laboratory, Los Alamos, NM 87545, U.S.A.; \\ ${ }^{\dagger}$ Department of Physics, University of Michigan, Ann Arbor, MI 48109-1120, U.S.A.; ${ }^{\ddagger}$ Stanford \\ Synchrotron Radiation Laboratory, Stanford University, Stanford, CA 94305, U.S.A.
}

(Received 4 April 1989 by H. Suhl)

\begin{abstract}
Resonant photoemission spectra of the $\mathrm{O} 2 p$-derived valence band of insulating $\mathrm{ThO}_{2}$ are compared to linear muffin-tin orbital (LMTO) density-of-state (DOS) and XPS intensity calculations. At Th $5 d$ corelevel threshold energies $(85 \leqslant h v \leqslant 120 \mathrm{eV})$, resonance is greatest at the bottom of the $\mathrm{O} 2 p$ band where calculated $p / d$ hybrid states are greatest; $p / f$ hybrid content is weak by comparison. We conclude that the dominant hybridization is between $\mathrm{O} 2 p$ states and Th $6 d$.
\end{abstract}

VALENCE BANDS in actinide materials have been the subject of numerous photoemission studies, both theoretical and experimental [1-12]. Resonant photoemission at the $5 d$ core-level threshold has proven to be a particularly effective probe for locating $5 f$ states in uranium compounds [6-10]. The classic example is uranium dioxide where strong Fano resonance is observed in occupied $5 f$ states near the Fermi level, $E_{F}$ $[7,9,10]$. The oxygen-derived $\mathrm{O} 2 p$ states in $\mathrm{UO}_{2}$ also resonate at photon energies near the $\mathrm{U} 5 d$ thresholds [9] in a process interpreted as signalling ligand-metal hybridization with greater $f$-hybrid content at the top of the band, i.e., at smaller binding energy, and correspondingly more $d$ at the bottom. With few exceptions, such resonant photoemission studies have been made on actinide materials such as uranium where the $5 f$ states are formally occupied.

In this report we describe valence-band resonance in insulating thoria, and present theoretical results relevant to understanding the spectra. The interesting aspect of the electronic structure of thoria is its simplicity in a purely ionic model: the Th states are formally depleted and only the $\mathrm{O} 2 p$ based band remains, inappropriate in describing actual bonding in the $\mathrm{ThO}_{2}$ where contribution of $5 f$ electrons to the $\mathrm{O} 2 p$ band is $c a .0 .6$ electron and the calculated charge on $\mathrm{Th}$ is +2.47 rather than the formal +4 . Hybridization becomes an important factor, but the relative magnitudes of $d$ and $f$ hybrid states may be reversed between $\mathrm{ThO}_{2}$ and $\mathrm{UO}_{2}$. Using relativistic potentials and Hartree-Fock calculations, Wadt [14] concluded that $5 f$ levels dominate back-bonding in the linear molecular ion, $\mathrm{UO}_{2}^{2+}$, whereas the $\mathrm{ThO}_{2}$ molecule is bent because of back-bonding with $6 d$. If this relative ordering of states is retained in the solid dioxides, $\mathrm{UO}_{2}$ and $\mathrm{ThO}_{2}$, then ligand- $d$ hybrid ground states would be more prominent in $\mathrm{ThO}_{2}$. We have performed LMTO calculations, presented below, which show that this difference between $\mathrm{UO}_{2}$ and $\mathrm{ThO}_{2}$ does indeed extend to the solid state.

Resonant photoemission processes of concern in thoria can be represented by two distinct cases: (a) within the purely ionic context without hybridization, and (b) with mixed ground-state configurations.

(a) Formal $\mathrm{Th}^{4+}, \mathrm{O}^{2-}$ atomic picture without hybridization:

$$
\underbrace{\frac{\text { ground state }}{5 d^{10} 5 f^{0} 6 d^{0}}}_{\mathrm{Th}^{4+}}
$$

a case which parallels $\mathrm{CeO}_{2}$ [13]. However, as Gubanov et al. [2] have pointed out on the basis of molecular cluster calculations, the term "formal valency" is

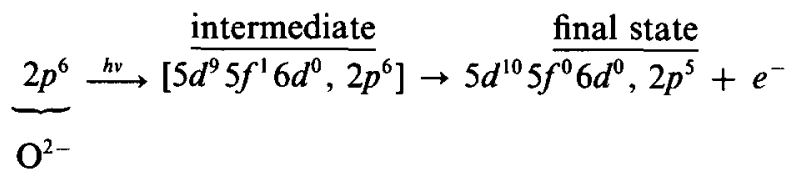

In this hypothetical case there is no resonant $5 f$ or $6 d$ emission since these ground states do not exist. 
(b) Adding hybridization:

scopic calculation exists for resonant valence-band

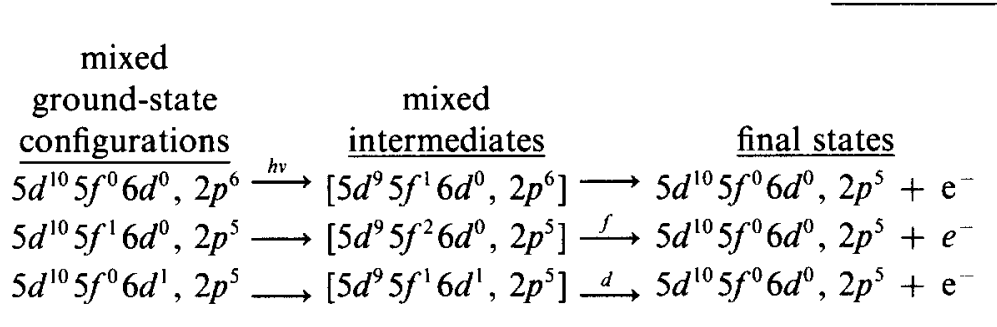

where $f$ means $\left\langle 5 f, 5 f\left|e^{2} / r\right| 5 d, e^{-}\right\rangle$and $d$ means $\langle 5 f$, $\left.6 d\left|e^{2} / r\right| 5 d, e^{-}\right\rangle$.

In all cases above, the final PES states are the same, but with hybridization there is resonance enhancement through the decay channels $f$ and $d$. Auger relaxation modes are added considerations discussed at the end of the article. Also, in direct photoemission at phonon energies $(h v)$ well below the $5 d$ binding energies $(\sim 100 \mathrm{eV})$, the intermediate states can be omitted. To date, we know of no thorough valence-band calculations for (a) and (b) above which include all of the excitations/relaxation modes in $\mathrm{ThO}_{2}$, and our discussions are to be considered within that context.

Spectral features in transition metal compounds can be understood only if such ligand-metal hybridization is accounted for as Davis makes clear in discussions of $3 d$ materials [15]. Cluster calculations on $\mathrm{NiO}$, for example, agree with bremsstrahlung isochromat spectroscopy (BIS) and X-ray photoelectron spectroscopy (XPS) and yield values for local interactions such as the $d-d$ Coulomb energy and ligand- $d$ hybridization [16]. Actinides may not be so tractable, however, especially in insulating $\mathrm{ThO}_{2}$ where the band gap is as wide as the bandwidth and cluster-type states, i.e., ligand-hole $/ f^{n}, d^{n}$ configurations, are unresolved.

In their theoretical treatment of the Th $4 f$ corelevel XPS spectra of actinide oxides [11] and thorium intermetallics [17] Gunnarsson et al. point out the crucial role played by differences in conduction-band profiles upon core-level satellite structures, and that the concepts of "well" and "poorly" screened final states make less sense for actinides than for the analogous rare-earth compounds [17] and that mixed states involving $f^{0}, f^{1}$, and $f^{2}$ character cannot be ignored. Using a generalized Anderson Hamiltonian for ThCo and $\mathrm{ThPt}_{3}$, they arrived at a $5 f$-conduction band hybridization parameter of $\Delta_{\mathrm{av}}=0.1 \mathrm{eV}$ and a core hole/ $5 f$ Coulomb interaction of $U_{f c} \sim 5 \mathrm{eV}$, a value not directly extractable from either XPS or BIS. In a point worth noting, they concluded that the correct interpretation of spectral features in actinides will have to rely heavily upon theoretical modeling. As far as we know, as stated above, no such detailed spectro- photoemission in thoria. Recognizing these points, we present our experimental PES results on $\mathrm{ThO}_{2}$ in the following, discuss them in relation to previous $\mathrm{UO}_{2}$ data [9], and compare with LMTO $p / d$ partial DOS and XPS calculations.

A $1 \mathrm{~cm}^{2} \times 0.2 \mathrm{~cm} \mathrm{ThO} \mathrm{C}_{2}\left(\begin{array}{lll}1 & 1 & 1\end{array}\right)$ wafer was cut from a single crystal boule, oriented to $\pm 1 / 2^{\circ}$ with Laue $\mathrm{X}$-ray diffraction, and lapped through progressively finer polishes to a mirror finish on $1 / 4-\mu \mathrm{m}$ diamond paste. A final chemical treatment in $\mathrm{HF}(\mathrm{g})$ at $186^{\circ} \mathrm{C}$ followed by successive rinses in dilute $\mathrm{HNO}_{3}, \mathrm{H}_{2} \mathrm{O}$ and ethanol removed the Beilby layer to a depth of $1 / 2 \mu \mathrm{m}$. Adsorbed gases were removed by heating $30 \mathrm{~min}$ at $960^{\circ} \mathrm{C}$ in a vacuum of $6 \times 10^{-11}$ torr $\left(8 \times 10^{-9} \mathrm{~Pa}\right)$, which produces a sharply defined $p(1 \times 1)$ LEED array with no remaining surface impurities detectable by Auger electron spectroscopy.

Photoemission data were taken in the photon energy range $80 \leqslant h v \leqslant 120 \mathrm{eV}$ on the $4^{\circ}$ Beamline 1-1 at the Stanford Synchrotron Radiation Laboratory (SSRL). Angle-integrated spectra, $42^{\circ} \pm 6^{\circ}$, were taken using a commerical CMA with a combined grating/analyzer resolution of $0.4 \mathrm{eV}$. Incident photon flux was monitored by the photoyield from a Ni wire mesh. The sample was stable with no changes in the spectra over several hours of beam exposure in this experiment; data were taken with the sample at room temperature. A commerical flood gun was used to minimize charging, but only energy distribution curves (EDCs) at fixed photon energies could be taken reliably. Spectra taken at different photon energies are compared by aligning the valence-band maxima (VBM) and assigning to them a relative binding energy of $E_{\mathrm{VBM}}=0$, as shown in Fig. 1 .

The Au Fermi level was also measured, and it lies about $2 \mathrm{eV}$ above the VBM of $\mathrm{ThO}_{2}$ and the vacuum level $4 \mathrm{eV}$ beyond.

Figure 1 shows data normalized to constant incident flux as taken at photon enegies below, at and above the Th $5 d$ core-level thresholds. Before discussing resonance effects, we point out the absence of structure in the band gap abve VBM. There are no surface states here and indeed none were expected from nearly stoichiometric thoria since fluoritic $\mathrm{ThO}$, 


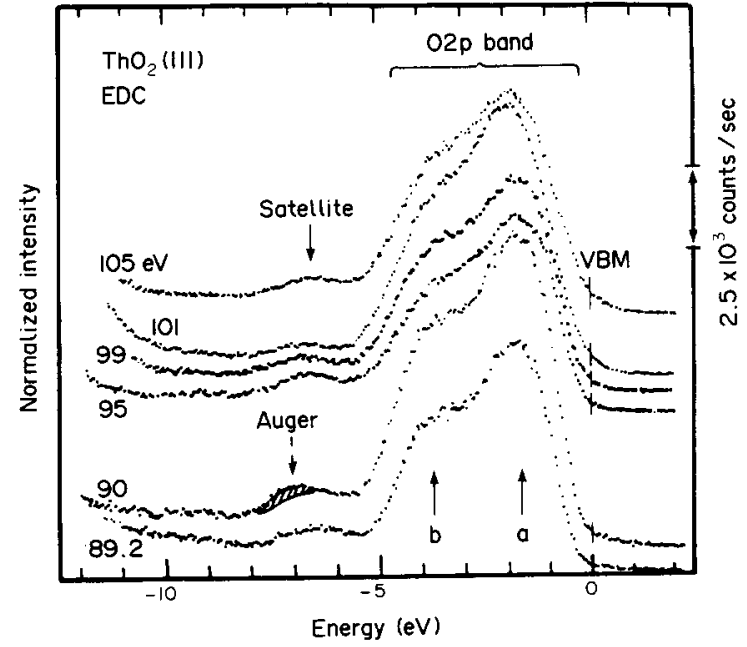

Fig. 1. Experimental EDC's for photon energies in the region of the Th $5 d \rightarrow 5 f$ edge. Binding energies are relative to the valence band maxima (VBM). Combined analyzer/grating resolution is $0.4 \mathrm{eV}$. Data normalized to constant incident flux,

(1 111$)$ is a charge neutral surface consisting of $\left(-\mathrm{O}_{-}\right.$ Th-O-) repeat layers terminating with oxygen as was the case with fluoritic $\mathrm{UO}_{2}\left(\begin{array}{lll}1 & 1 & 1\end{array}\right)$ [18].

In Fig. 1 a sharp increase in intensity is seen upon changing $h v$ from 89.2 to $90.0 \mathrm{eV}$, a minimum occurs at $95 \mathrm{eV}$ and another maximum at $101 \mathrm{eV}$. Peak-height intensities derived from Fig. 1 are plotted in Fig. 2. The first maximum at $h v \sim 90 \mathrm{eV}$ is very sharp with a full width of less than $2 \mathrm{eV}$; the second maximum at $\sim 101 \mathrm{eV}$ is somewhat broader. These peaks are principally of atomic origin and result from the Th-derived

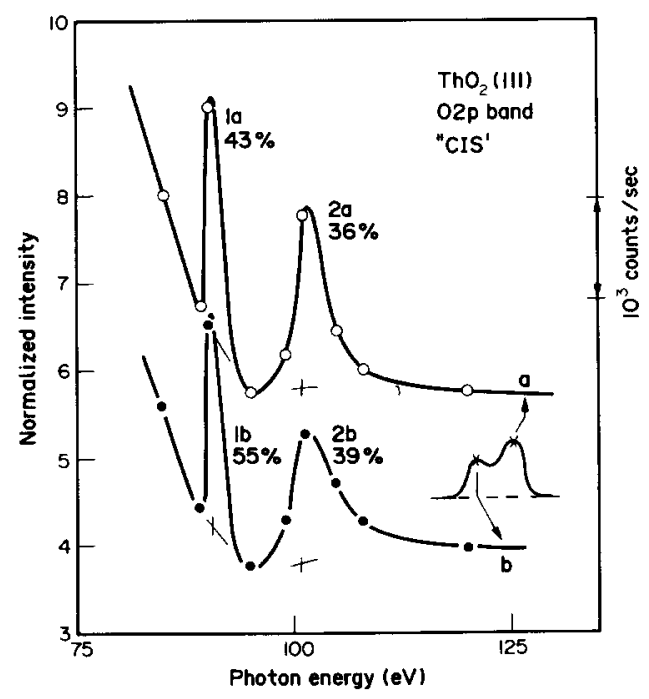

Fig. 2. Derived constant initial state (CIS) curves for the upper maximum at " $a$ " and the lower maximum at " $b$ ". Points were taken from the data files of Fig. 1.
${ }^{3} D(90 \mathrm{eV})$ and ${ }^{1} P(101 \mathrm{eV}), 5 d^{10} 5 f^{0} \rightarrow\left[5 d^{9} 5 f^{1}\right]$, ground state to intermediate multiplet excitations [19] (see below). Within a fraction of an $\mathrm{eV}$, the same maxima were observed in $\mathrm{Th}$ metal [19] and in $\mathrm{Th}_{2} \mathrm{Zn}_{17}$ [20]. The peaks in Fig. 2 are numbered and the percentage increases in peak heights are given. Figure 2 is significant on several counts. First, not only does the $\mathrm{O} 2 p$ band resonate at the Th $5 d$ thresholds in the absence of the "spectator" $5 f$ states near $E_{F}$ present in $\mathrm{UO}_{2}$ [9], but the magnitude of resonance is as great as in the $\mathrm{O}$ $2 p$ band of $\mathrm{UO}_{2}$ where the corresponding percentages are: $1 \mathrm{a}-47 \%, 2 \mathrm{a}-60 \%, 1 \mathrm{~b}-39 \%$, and $2 \mathrm{~b}-$ $55 \%$. The resonance peaks in $\mathrm{UO}_{2}$ are broader with more structure as a result of the much larger number of $5 d^{10} 5 f^{2} \rightarrow\left[5 d^{9} 5 f^{3}\right]$ excitations. With atomic $\mathrm{Th}$ there are only three transitions to consider with calculated energies and relative dipole oscillator strengths of ${ }^{3} P(86.1 \mathrm{eV})-0.0071,{ }^{3} D(90.8 \mathrm{eV})-$ 0.4967 , and ${ }^{1} P(105.2 \mathrm{eV})-12.4124$. These atomic photo-absorption calculations do not include solidstate effects or subsequent decay cross sections including autoionization, (e.g, decay in (a) above), factors which are important because they alter the relative strengths of the ${ }^{3} D$ and ${ }^{1} P$ resonances and shift the energies. The ${ }^{3} P$ transition is too weak to be observed.

Figure 2 demonstrates ligand-metal coupling in thorium dioxide. Significantly, opposite trends are observed in the resonance behaviour of $\mathrm{UO}_{2}$ and $\mathrm{ThO}_{2}$. In $\mathrm{UO}_{2}$ the top of the $\mathrm{O} 2 p$ band resonates more strongly than the bottom, and at the higher photon energy line, the resonance is greater [9]; Fig. 2 shows the reverse in $\mathrm{ThO}_{2}$. Although the $\mathrm{O} 2 p$ bands in $\mathrm{ThO}_{2}$ and $\mathrm{UO}_{2}$ look almost identical and both resonate at the actinide $5 d$ thresholds, they are different on closer inspection. This difference implies that the $5 f / 5 d$ hybrid roles in the $\mathrm{O} 2 p$ states in $\mathrm{ThO}_{2}$ and $\mathrm{UO}_{2}$ are reversed, in agreement with Wadt's calculations on isolated molecules [14]. To pursue this matter further,

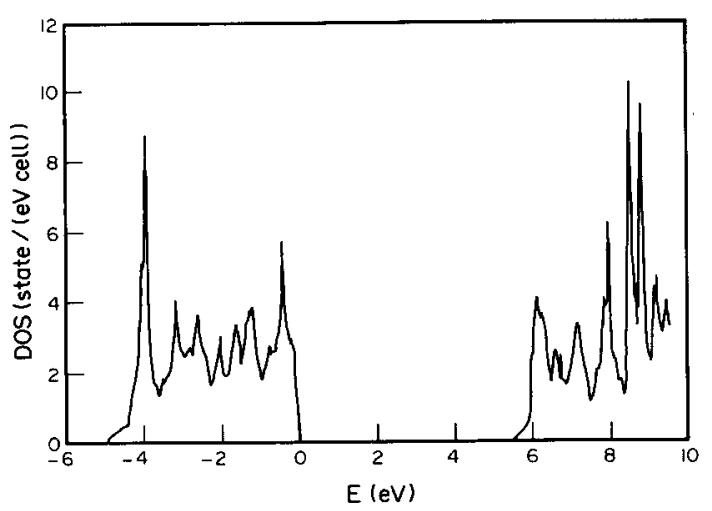

Fig. 3. Total DOS curves for $\mathrm{ThO}_{2}$ showing the oxygen derived valence bands and the conduction bands. $E_{\mathrm{VBM}}$ is at zero. 


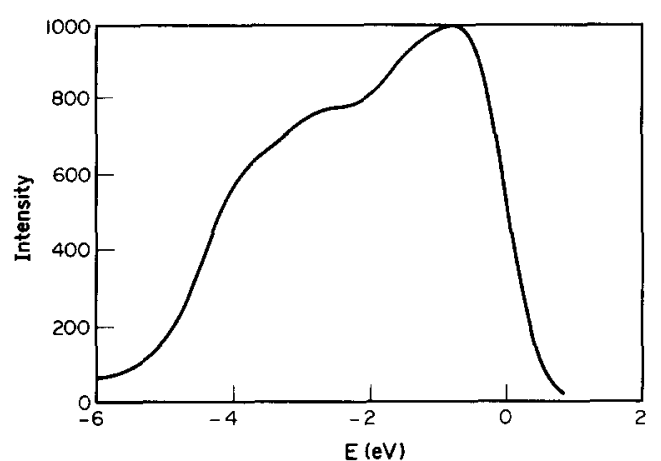

Fig. 4. Total calculated XPS intensity (at $200 \mathrm{EV}$ incident photon energy). $E_{\mathrm{VBM}}$ is at zero.

we performed linear-muffin-tin orbital (LMTO) band calculations [21] on $\mathrm{ThO}_{2}$.

Figure 3 summarizes the ground-state LMTO calculations. Several features are to be noted. First, the band gap of $E_{\text {gap }} \sim 5 \mathrm{eV}$ is approximately correct for this transparent, colourless, and insulating oxide. Also, the calculated bandwidth of $5 \mathrm{eV}$ agrees with experiment. Second, there is more structure in Fig. 3 than observed experimentally in Fig. 1, signaling lifetime and resolution broadening in the data. Also in Fig. 3 it is seen that there is a large peak in the DOS at the bottom of the $O 2 p$ band not seen in the experimental data. In Fig. 4 the total calculated XPS intensity at $200 \mathrm{eV}$ incident photon energy is given. This result is obtained by multiplying the DOS function of Fig. 3 by the calculated cross-sections and modifying that by lifetime broadening $(1.0 \mathrm{eV})$ and instrumental resolution $(0.45 \mathrm{eV})$ [22]. (The cross-sections were calculated at $200 \mathrm{eV}$ instead of $100 \mathrm{eV}$ because we do not believe the approximations made in this calculation are valid at $100 \mathrm{eV}$ - see Ref [22]). As can be seen there is reasonably good agreement between the calculated and measured XPS intensity of the $\mathrm{O} 2 p$ band. To explain the difference between the resonant behaviour of $\mathrm{UO}_{2}$ and $\mathrm{ThO}_{2}$ the $l=2$ and $l=3$ Th crosssections at $200 \mathrm{eV}$ incident photon energy are shown in Fig. 5. Here the ratio of the $l=3$ to $l=2$ crosssections is about 0.1 . To compare with $\mathrm{UO}_{2}$, for which we have no cross-section results, we did the following. We calculated this ratio in $\mathrm{ThO}_{2}$ at $1486 \mathrm{eV}$ incident photon energy obtaining a value of $\sim 0.7$. From crosssection calculations on other $U$ compounds (see Ref. [23]) at this incident energy we estimated this ratio to be between 5 and 7 in $\mathrm{UO}_{2}$. We also estimated that the $5 f$ electron count in the $\mathrm{O} 2 p$ band in $\mathrm{UO}_{2}$ (unpublished LAPW results) is slightly larger than in $\mathrm{ThO}_{2}$ (present LMTO results). Therefore the contribution to the XPS intensity from the $d$ states is stronger in $\mathrm{ThO}_{2}$ than in $\mathrm{UO}_{2}$. The $\mathrm{ThO}_{2}$ results are shown in Fig. 6 and it is seen that indeed the calculated XPS intensity

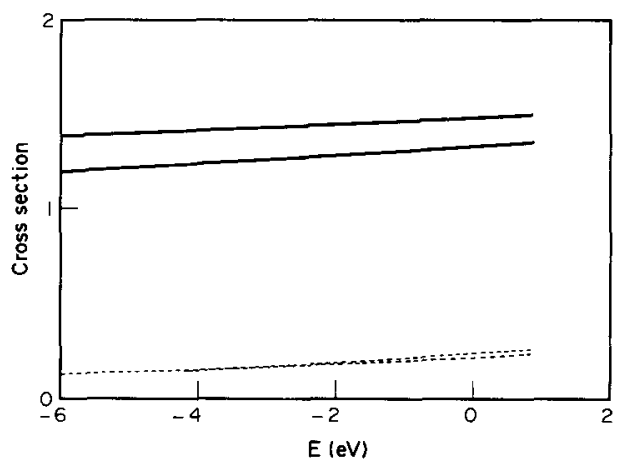

Fig. 5. Cross-sections for the Th $l=2$ (solid lines) and $l=3$ (dashed lines) which include spin-orbit splitting.

$l=2$ contribution is an order of magnitude larger than the $l=3$ contribution. In $\mathrm{UO}_{2}$ the relative intensities of calculated $d$ and $f$ states in the $\mathrm{O} 2 p$ band should be reversed from $\mathrm{ThO}_{2}$, but in both oxides $f$ hybrids are at the top and $d$ hybrids mainly at the bottom of the band. Resonance in $\mathrm{UO}_{2}$ signaled dominant $p / f$ hybrids at the top. But with $\mathrm{ThO}_{2}, f$ state cross-sections are much weaker and the difference in resonance between $\mathrm{UO}_{2}$ and $\mathrm{ThO}_{2}$ correlates with larger $p / d$ hybrid contribution at the bottom of the $\mathrm{O}$ $2 p$ band in $\mathrm{ThO}_{2}$.
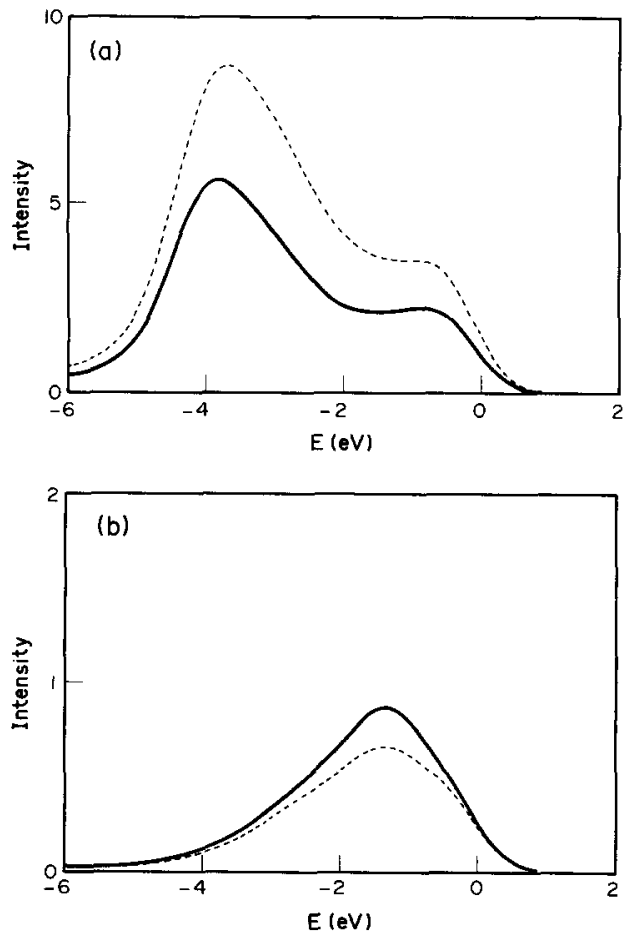

Fig. 6. (a) Th intensities (at $200 \mathrm{eV}$ ) showing both spin-orbit components of the $l=2$ channel; (b) Th intensities (at $200 \mathrm{eV}$ ) showing both spin-orbit components of the $l=3$ channel. 
Before summarizing, we discuss one other feature of our data. For Th metal [19] Auger decay was identified and for intermetallic compounds of $U$ it has been proposed that this decay channel can mask the $5 f$ partial density of states [24], a contention whose general validity has been disputed [25]. In the case of $\mathrm{ThO}_{2}$, as seen in Fig. 1, Auger features are mostly negligible with only the weak $\mathrm{O}_{5} V V$ transition appearing at the ${ }^{3} D$ threshold. There is no evidence of the $\mathrm{O}_{4} V V$ excitation at the ${ }^{\prime} P$ energy and above and although autoionization remains a consideration, it alone does not appear to account for the difference between $\mathrm{ThO}_{2}$ and $\mathrm{UO}_{2}$. Unfortunately, the $\mathrm{O}_{5} V V$ Auger transition overlaps the satellite and masks its resonance at $90 \mathrm{eV}$. This satellite is considered important because it relates to the presence of both initial and final-state configurations as discussed above [17]. At photon energies where there is no interference, the satellite variation in Fig. 1 is opposite to that of the O $2 p$ band: at $h v=101 \mathrm{eV}$ the satellite is about onehalf as intense as at $95 \mathrm{eV}$, whereas in $\mathrm{UO}_{2}$ the weak satellite resonated in phase with everything else. Further investigation into these intriguing subtleties must await detailed spectroscopic calculations as advised by Gunnarsson et al. [17].

In summary, we have compared resonant photoemission in the valence band of $\mathrm{ThO}_{2}$ with LMTO DOS and XPS intensity calculations. We find that the dominant contribution is due to the hybridization between $\mathrm{O} 2 p$ and Th $6 d$ at the bottom of the $\mathrm{O} 2 p$ band.

Acknowledgements - We thank K. Shung for helpful discussions. The Stanford Synchrotron Radiation Laboratory and the work at Los Alamos National Laboratory are supported by the U.S. Department of Energy. Research support by the U.S. National Science Foundation-Low Temperature Physics Program, Grand No. DMR-87-21654 (J.W.A.) is gratefully acknowledged.

\section{REFERENCES}

1. B.W. Veal \& D.J. Lam, Phys. Rev. B10, 4902 (1974).

2. V.A. Gubanov, A. Rosén \& D.E. Ellis, Solid State Commun. 22, 219 (1977).

3. G.C. Allen, P.M. Tucker \& J.W. Tyler, Phil. Mag. B48, 63 (1983).
4. Y. Baer \& J. Schoenes, Solid State Commun. 33, 885 (1980).

5. L.E. Cox, J. Electron Spectrosc. Relat. Phenom. 26, 167 (1982).

6. M. Iwan, E.E. Koch \& F.-J. Himpsel, Phys. Rev. B24, 613 (1981).

7. B. Reihl, N. Martensson, D.E. Eastman, A.J. Arko \& O. Vogt, Phys. Rev. B26, 1842 (1982).

8. J.W. Allen, S.-J. Oh, L.E. Cox, W.P. Ellis, M.S. Wire, Z. Fisk, J.L. Smith, B.B. Pate, I. Lindau \& A.J. Arko, Phys. Rev. Lett. 54, 2635 (1985).

9. L.E. Cox, W.P. Ellis, R.D. Cowan, J.W. Allen, S.-J. Oh, I. Lindau, B. B. Pate \& A.J. Arko, Phys. Rev. B35, 5761 (1987).

10. A.J. Arko, D.D. Koelling, A.M. Boring, W.P. Ellis \& L.E. Cox, J. Less-Common Met. 122, 95 (1986).

11. O. Gunnarson, D.D. Sarma, F.U. Hillebrecht \& K. Schönhammer, J. Appl. Phys. 63, 3676 (1988).

12. A. Zangwill \& D.A. Liberman, Phys. Rev. B36, 6705 (1987).

13. D.D. Koelling, A.M. Boring \& J.H. Wood, Solid State Commun. 47, 227 (1983); J.W. Allen, J. Magn. Magn. Mater. 47, 168 (1985).

14. W.R. Wadt, J. Amer. Chem. Soc. 103, 6053 (1981).

15. L.C. Davis, Phys. Rev. B25, 2912 (1982).

16. G.A. Sawatzky \& J. W. Allen, Phys. Rev. Lett. 53, 2339 (1984).

17. O. Gunnarsson, K. Schönhammer, D.D. Sarma, F.U. Hillebrecht \& M. Compagna, Phys. Rev. B32, 5499 (1985).

18. W.P. Ellis \& T.N. Taylor, Surf. Sci. 91, 408 (1980).

19. A. Fujimori \& J.H. Weaver, Phys. Rev. B31, 6411 (1985).

20. Y. Laissailly, J.W. Allen, W. Ellis, L. Cox, B. Pate, Z. Fisk \& I. Lindau, J. Magn. Magn. Mater. (to appear).

21. H.L. Skriver, The LMTO Method, SpringerVerlag, Berlin (1984).

22. P. Marksteiner, P. Weinberger, R.C. Albers, A.M. Boring \& G. Schadler, Phys. Rev. B34, 6730 (1986).

23. A.M. Boring, R.C. Albers, G. Schadler, P. Marksteiner \& P. Weinberger, Phys. Rev. B35, 2447, (1987). This paper shows the notes of the $\mathrm{U} 5 F / 6 d$ cross-section in $\mathrm{UBe}_{13}$ which should be similar to those in $\mathrm{UO}_{2}$.

24. D.D. Sharma, F.U. Hillebrecht, C. Carbone \& A. Zangwill, Phys. Rev. B36, 2916 (1987).

25. A.J. Arko, D.D. Koelling, C. Capasso, M. del Giudice \& C.G. Olsen, Phys. Rev. B (in press). 